\title{
Integration of the Supplier Capacity for Choosing the Less Risky Schedule within an Uncertain Environment
}

\author{
Romain Guillaume ${ }^{1,2}$, Caroline Thierry ${ }^{1}$, and Bernard Grabot ${ }^{2}$ \\ ${ }^{1}$ Université de Toulouse IRIT / UTM 5, Allées A. Machado, \\ 31058 Toulouse Cedex 1, France \\ guillaumairit.fr, thierry@univ-tlse2 \\ ${ }^{2}$ Université de Toulouse INPT / LGP / ENIT 47 Av. d'Azereix, \\ BP 162965016 Tarbes Cedex, France \\ benardeenit.fr
}

\begin{abstract}
This communication addresses the integration of the supplier capacity in the procurement planning process of a customer within a supply chain. Since this supply chain evolves within an uncertain environment, uncertainties may be due to an ill-known demand (prevision) and to the customer production system (scraps, imprecise processing time...). Thus, we propose a collaborative process where the customer aims at taking the less risked decision. The integration of the supplier capacity in the gross requirement enables to assess the risk of back order so that the feasibility of the requirement plan. It then enables the customer to make the decisions which limit this risk.
\end{abstract}

Keywords: Planning, Decision under uncertainty, Possibility theory, Supply chain.

\section{Introduction}

Nowadays, companies are not competing as independent entities but as a part of collaborative supply chains. The uncertainty on the demand creates risk in the supply chain as backordering, obsolete inventory due to the bullwhip effect. To reduces this risque to different approche exist the collaboration between customer and suplier or the integration of the uncertainty into the planning process.

The collaborative processes mainly aim at reducing the supply risk [1]. The coordination of the supply chain can be realized by two different ways: the vertical and horizontal approach. The vertical is a centralized decision making which synchronize the supply chain (common way to coordinate within companies). The horizontal approach is referred to the collaborative planning (supply chain of independent entities) [2]. The industrial collaborative process has been standardized for implementing cooperation between retailers and manufactures. This process is called "Collaborative Planning, Forecasting and Replenishment" (CPFR®) [3].

These techniques aim at creating short and reactive decision loops between customers and suppliers in order to cope with the growing uncertainty on demand forecasting, due to the shortening of the product life cycle and to customers' versatility. 
Within these supply chains, the collaborative processes are usually characterised by a set of point-to-point (customer / supplier) relationships with partial information sharing [1].

More precisely, a procurement plan, or several procurement plans, are built and propagated through the supply chain. Taking into account the uncertainty of the demand in the planning processes itself can be another solution for making more "informed decisions" [4][5][6]. And then integrate the uncertainty into the cooperative planning processes with the suppliers, what we propose in this article.

Within this context, this communication focuses on the integration of imperfection on the customer's gross requirements, taking into account his knowledge on the capacity of the suppliers, with the final goal to cope with an uncertain environment (linked to demand, scraps, processing time...)...

The theory of possibility and the theory of fuzzy sets [7][8] are often used to model the uncertainty when historical data can hardly be obtained or are obsolete [9][10][11]. Different models have been used to represent the imperfection on the requirements. [6] represents the imprecision on the quantity by a trapezoidal fuzzy number; [5] integrates the uncertainty on the order and the imprecision on the quantity to compute the demand.

In a previous work (see [12]), we consider the uncertainty on the order itself, so that the imprecision and the uncertainty on the quantity and on the date. Moreover, the dependencies between the requirements are considered (see [4]) to compute a gross requirement, modelled by a graph. This graph has possibility levels on arcs and fuzzy quantities on nodes.

In this communication, we propose a method to integrate the supplier capacity before choosing the final procurement plan and the associated production sequence. This method is composed of three steps: 1) design of the graph which represents all possible gross requirements (section 2), 2) computation of the level of feasibility of each node (section 3), 3) choice of the final schedule (section 4).

\section{Gross Requirement}

In this section is first introduced the model of requirements and dependencies, followed by the method to design a graph representing these possible requirements.

\subsection{Model of Requirements}

We consider that the customer has a make-to-Order production. In this context, the requirements of components are expressed by a quantity for a date which can be both imperfect (uncertain and/or imprecise). Moreover, the requirement itself can be uncertain (i.e. may be cancelled). Possibility theory is suggested to represent these imperfections. The requirement is thus composed by two sets of fuzzy sets: the first one represents the imperfect quantities and the uncertainty on the requirement, whereas the second one represents the imperfectly known date. For instance, Figure 1 represents an imperfect requirement (this model is developed in [12] and [13]). 

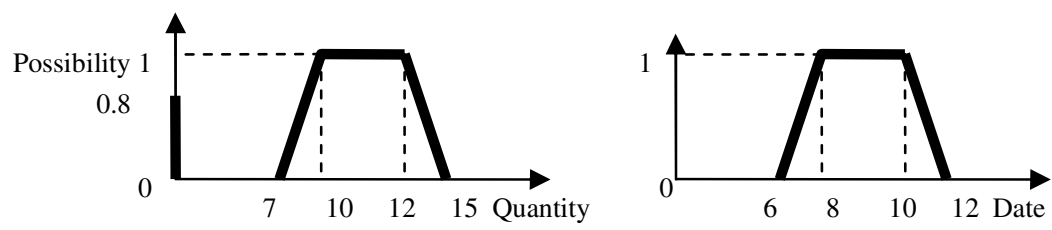

Fig. 1. Uncertain requirement (quantity equal at zero possible) with an imprecision on the quantity and an uncertainty and imprecision on the date

Dependencies often exist between requirements. These dependencies are linked to constraints linked to the bills of materials or to the resources (assembly lines) (see [10]). The first kind of dependency (denoted "quantity dependencies") exists if the required component appears at different levels in the bill of material of the same final product (see Figure 2).
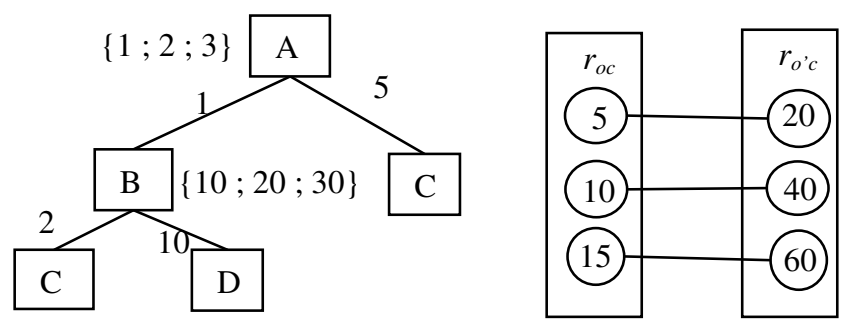

Fig. 2. Quantity dependencies; the requirement is linked with the requirement $r_{o^{\prime} c}$ by the component $\mathrm{B}$. The possible quantities of $\mathrm{r}_{\mathrm{oc}}$ are linked with the possible quantities of $\mathrm{r}_{\mathrm{o}^{\prime} \mathrm{c}}$. When $\mathrm{r}_{\mathrm{oc}}$ is equal at 5 then $r_{o^{\prime}}$ is equal to 20 (similarly, 10 with 40 and 15 with 60).

On the other hand, requirements processed on the same assembly line can be linked by "precedence dependencies" (see Figure 3).

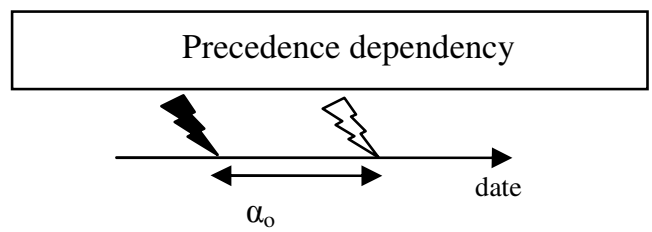

Fig. 3. Illustration of a "precedence dependency" between two requirements: the requirement $r_{o}$ (black) and the requirement $r_{o}$, (white)

Moreover, the customer may consider some feasible sequences evaluated with a level of preference (see Figure 4). 

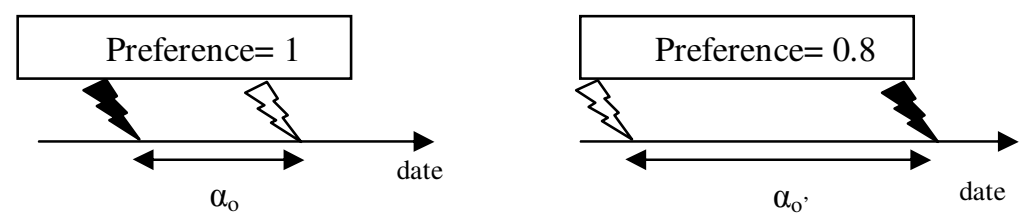

Fig. 4. Possible sequences, between two requirements: requirement $r_{o}$ (black) and requirement $r_{o}$ (white)

\subsection{Gross Requirement Model}

Since the supplier is supposed to perform a Make-to-Stock production, he has to compute gross requirement expressed as quantities by period from requirements expressed as quantities for date (due to make to his order production) in order to build a requirement planning.

From the information on the requirements, the dependencies and the levels of preference between two decisions, a graph which represents the gross requirements can be built. The method to design this graph is explicitly explained in [4]: the nodes $\left(t, c_{t}\right)$ of the graph represent the possible quantities (represented by a trapezoidal fuzzy number) for each given period) (see Figure 5). The $\operatorname{arcs}\left(t, c_{t}, c_{t+1}\right)$ of the graph are valued by a set of characteristics $A_{t, c_{t}, c_{t+1}}$ : a possibility level and the preference linked to a given quantity for period $t$ and a given quantity for period $t+1$. So, the graph (see Figure 6) takes into account the imperfections on the requirements, the dependencies between requirements and the preferences on the chosen sequences.

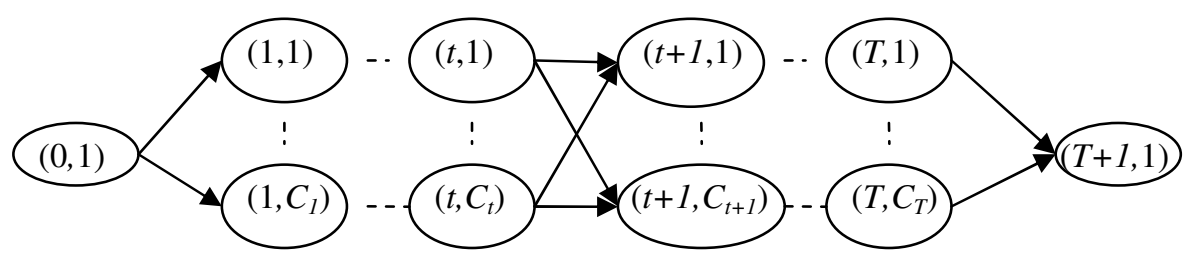

Fig. 5. Representation of gross requirements

Notations:

$t$ : period with $t \in[1, \mathrm{~T}]$

$c_{t}$ : index of fuzzy gross required quantity of period $t$ with $c_{t} \in\left[1, \mathrm{C}_{\mathrm{t}}\right]$

$s:$ index of the sequence with $s \in[1, \mathrm{~S}]$

$k_{s}$ : index on the choice of sequence $s$ with $k_{s} \in\left[1, \mathrm{~K}_{\mathrm{s}}\right]$

$\tilde{D}_{t, c_{t}}$ : fuzzy gross required quantity on node $\left(t, c_{t}\right)$

$A_{c_{t}, c_{t+1}, t}$ : set of characteristics of the arc $\left(t, c_{t}, c_{t+1}\right)$ linking the node $\left(t, c_{t}\right)$ with the node $\left(t+1, c_{t+1}\right)$ 
$w_{c_{t}, c_{t+1}, t}$ : possibility level of the arc linking the node $\left(t, c_{t}\right)$ with the node $\left(t+1, c_{t+1}\right)$

$\mu_{s, k_{s}}$ : preference level of a sequence $s$ for the choice $k_{s}$

The set $A_{c_{t}, c_{t+1}, t}$ is defined by $A_{c_{t}, c_{t+1}, t}=\left\{w_{c_{t}, c_{t+1}, t} ; \mu_{1, k_{1}} ; \ldots ; \mu_{S, k_{S}}\right\}$.

\section{Computation of Feasibility Level}

This section addresses the computation of the feasibility of the graph by considering the delivering constraints of the supplier. These constraints are ill-known. The delivering capacity of the supplier can be transmitted by the supplier himself. The customer may also have his own knowledge on these constraints (in this study we focus on the computation of the level of feasibility and not on the problem of confidentiality of data and degree of collaboration between the customer and the supplier). The supply constraints are modeled by triangular fuzzy number (see Figure 7). To compute the level of feasibility of the gross requirements, the inventories or backorders are considered. Thus, we evaluate the necessity to satisfy the gross requirements with the possible delivered quantity (production plus inventories minus backorders).

\section{Notations:}

input data:

$\widetilde{C}_{t}$ : maximum supply capacity of the period $t$

$\tilde{D}_{t, c_{t}}:$ gross required quantity $\left(t, c_{t}\right)$

$w_{c_{t}, c_{t+1}, t}$ : possibility level of the arc linking the node $\left(t, c_{t}\right)$ with the node $\left(t+1, c_{t+1}\right)$

\section{Dependent parameters:}

$\tilde{I}_{t, c_{t}}^{\prime}$ : possible inventory which can be positive or negative, for the node $\left(t, c_{t}\right)$

$\widetilde{I}_{t, c_{t}}$ : inventory of the node $\left(t, c_{t}\right)$

$\widetilde{B}_{t, c_{t}}$ : backorders of the node $\left(t, c_{t}\right)$

\section{Ouput data:}

$\Phi_{t, i}$ : level of feasibility of the gross required quantity $\left(t, c_{t}\right)$

The fuzzy numbers $\tilde{F}$ are expressed as quadruplets $\left(a_{F} ; b_{F} ; c_{F} ; d_{F}\right)$. The level of feasibility is computed using algorithm 1 . The possible inventory level of the previous nodes is firstly computed, then the feasibility of the nodes is assessed and the critical general fuzzy inventory is deduced. Finally, the fuzzy inventory and the fuzzy backorder levels are estimated. 
Algorithm 1. Computation of feasibility level

Input: Graph $G=<V, A>$, and fuzzy supplier Capacity $\widetilde{C}_{t}$;

Output: level of feasibility of each node $\Phi_{t, c_{t}}$;

\section{For each $t$ do}

For each note $\left(t, c_{t}\right)$ do

For each $c_{t-1} \mid w_{c_{t-1}, c_{t}, t-1} \neq 0$ do

//computation of the possible inventory level of the previous nodes//

If $\widetilde{B}_{t-1, c_{t-1}}=0$

$$
\begin{aligned}
& \text { Then } \tilde{I}_{t, c_{t}, c_{t-1}}^{\prime}=\widetilde{I}_{t-1, c_{t-1}}(+) \widetilde{C}_{t}(-) \tilde{D}_{t, c_{t}} ; \\
& \text { Else } \tilde{I}_{t, c_{t}, c_{t-1}}^{\prime}=\widetilde{C}_{t}(-) \widetilde{B}_{t-1, c_{t-1}}(-) \widetilde{D}_{t, c_{t}} ;
\end{aligned}
$$

\section{End if}

\section{End for each}

//computation of the feasibility of the nodes//

$$
\Phi_{t, c_{t}}=\min _{c_{t-1}}\left(\Phi_{t, c_{t}, c_{t-1}}\right)=\min _{c_{t-1} \mid w_{c_{t-1}, c_{t}, t-1} \neq 0}\left(\left(1-\mathrm{N}\left(\tilde{I}_{t, c_{t}, c_{t-1}}^{\prime} \geq 0\right)\right) \times w_{c_{t-1}, c_{t}}\right) ;
$$

//computation of the critical fuzzy general inventory level//

$$
\tilde{I}_{t, c_{t}}^{\prime}=\tilde{I}_{t, c_{t}, c_{t-1}}^{\prime} \mid \Phi_{t, c_{t}}=\Phi_{t, c_{t}, c_{t-1}} ;
$$

//computation of the fuzzy inventory and fuzzy backorder levels//

$$
\begin{aligned}
& \tilde{I}_{t, c_{t}}=\left\{\begin{array}{l}
a_{\tilde{I}_{t, c_{t}}}=\max \left(0 ; a_{\tilde{I}_{t, c_{t}}^{\prime}}\right) ; b_{\tilde{I}_{t, c_{t}}}=\max \left(0 ; b_{\tilde{I}_{t, c_{t}}}\right) \\
c_{\tilde{I}_{t, c_{t}}}=\max \left(0 ; c_{\tilde{I}_{t, c_{t}}^{\prime}}\right) ; d_{\tilde{I}_{t, c_{t}}}=\max \left(0 ; d_{\tilde{I}_{t, c_{t}}^{\prime}}\right)
\end{array}\right\} ; \\
& \tilde{B}_{t, c_{t}}=\left\{\begin{array}{l}
a_{\tilde{B}_{t, c_{t}}}=\max \left(0 ;-a_{\tilde{I}_{t, c_{t}}}\right) ; b_{\widetilde{B}_{t, c_{t}}}=\max \left(0 ;-b_{\tilde{I}_{t, c_{t}}^{\prime}}\right) \\
c_{\widetilde{B}_{t, c_{t}}}=\max \left(0 ;-c_{\tilde{I}_{t, c_{t}}^{\prime}}\right) ; d_{\tilde{B}_{t, c_{t}}}=\max \left(0 ;-d_{\tilde{I}_{t, c_{t}}^{\prime}}\right)
\end{array}\right\} ;
\end{aligned}
$$

\section{End for each}

\section{End for each}

$\operatorname{Return} \Phi_{t, c_{t}}$;

\section{Choice of the Manufacturing Sequence}

From the feasibility level and the knowledge on the manufacturing sequences, the "risk" $r_{s, k_{s}}$ linked to the choice $k_{s}$ for the sequence $s$ (equation (1)) is computed. The choice $K_{s}$ is made, which maximizes the preference level in the set of the less "risky" decisions (equation (2)). 

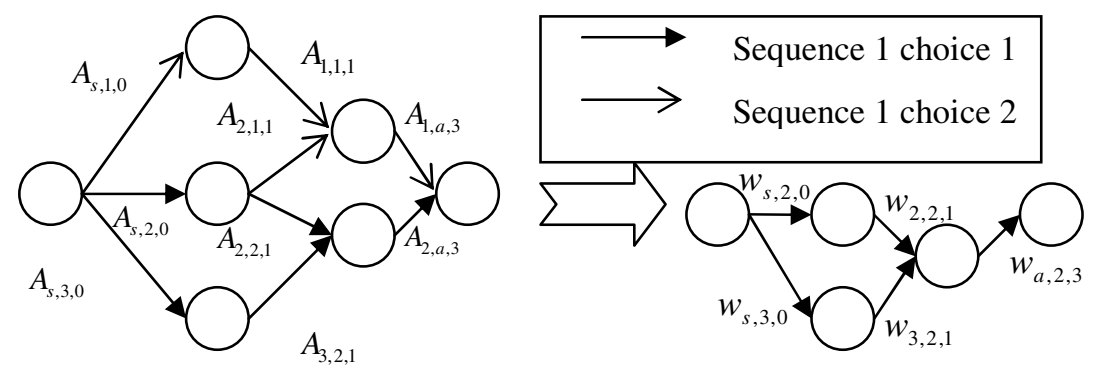

Fig. 6. Representation of graph before and after the integration of supplier capacity. The graph has two possible choices for one sequence (left graph). The decision is taken and the resulting graph is on the right side of the figure (choice 1 has been selected).

The preferences between two sequences are considered as less important than the "risk". Therefore, the preferences are used for the choice between two sequences of equivalent risk. After making all the choices on the sequences, the graph is reduced. The characteristics of the arcs $\left(t,, c_{t}, c_{t+1}\right) A_{t, c_{t}, c_{t+1}}$ are reduced to the possibility level $w_{c_{t}, c_{t+1}, t}$. Figure 7 shows an example of graph before and after decision. In the initial graph (before the decision), there are two possible choices for one sequence. After the decision (choice 1), the resulted graph is obtained.

$$
\begin{gathered}
r_{s, k}=\max _{t, c_{t} \mid \mu_{s, k_{s}} \in A_{c_{t-1}, c_{t}, t-1}}\left(1-\Phi_{t, c_{t}}\right) . \\
K_{s}=k_{s} \mid \max _{k_{s} \mid r_{s, k_{s}}=\min _{k_{s}}\left(r_{s, k_{s}}\right)}\left(\mu_{s, k_{s}}\right) .
\end{gathered}
$$

\section{Conclusion}

In this paper, a model of gross requirement plan which takes into account the imperfections on the requirements, the dependencies between requirements and the possible manufacturing sequences has been presented and the Delivering capacity of the supplier. A method allowing to compute the feasibility of each sequence, then to choose a sequence has been proposed. This method allows the customer to choose the less risky sequence in terms of backorder.

In collaborative process the customer and the supplier could negotiate on the delivering capacity, the risk level and a price. In fact, if the risk of the optimal solution is too high the customer can ask the supplier to propose a other delivering constrains (of course with a cost). 
Acknowledgments. This study has been performed with the support of Région MidiPyrénées and of the University of Toulouse.

\section{References}

1. Galasso, F., Mercé, C., Grabot, B.: Decision support for supply chain planning under uncertainty. In: 12th IFAC International Symposium Information Control Problems in Manufacturing (INCOM), St-Etienne, France, pp. 233-238 (2006)

2. Dudek, G.: Collaborative planning in Supply Chains. Lecture notes in economics and mathematical systems, pp. 533 (2004)

3. Ireland, R., Crum, C.: Supply chain Collaboration: how to implement CPFR® and other best collaborative practices. Integrated Business Management series and APICS (2005)

4. Guillaume, R., Thierry, C., Grabot, B.: Integration of ill-known requirements with dependencies into a gross requirement plan. In: 8ème ENIM IFAC Conférence Internationale de Modélisation et Simulation, Hammamet, Tunisia, May 10-12 (2010)

5. Grabot, B., Geneste, L., Reynoso Castillo, G., Vérot, S.: Integration of uncertain and imprecise orders in the MRP method. Journal of Intelligent Manufacturing 16(2), 215-234 (2005)

6. Fargier, H., Thierry, C.: The use of Possibilistic Decision Theory in Manufacturing, Planning and Control: Recent Results in Fuzzy Master Production Scheduling. In: Slowinski, R., Hapke, M. (eds.). Studies in fuzziness and soft computing, vol. 36, pp. 45-59. Springer, Heidelberg (2000)

7. Zadeh, L.A.: Fuzzy sets as a basis for a theory of possibility. Fuzzy Sets and Systems 1, 3-28 (1978)

8. Dubois, D., Prade, H.: Possibility Theory. Plenum Press, New York (1988)

9. Peidro, D., Mula, J., Poler, R., Verdegay, J.L.: Fuzzy optimization for supply chain planning under supply, demand and process uncertainties. Fuzzy Sets and Systems 160(18), 2640-2657 (2009)

10. Lan, Y.F., Liu, Y.K., Sun, G.J.: Modeling fuzzy multi-period production planning and sourcing problem with credibility service levels. Journal of Computational and Applied Mathematics 231(1), 208-221 (2009)

11. Aliev, R.A., Fazlollahi, B., Guirimov, B.G., Aliev, R.R.: Fuzzy-genetic approach to aggregate production-distribution planning in supply chain management. Information Sciences 177(20), 4241-4255 (2007)

12. Guillaume, R., Thierry, C., Grabot, B.: Intégration de besoins en composants mal connus dans un plan. In: 8ème Congrès International de Génie Industriel (CIGI 2009), Bagnères de Bigorre, France, June 10-12 (2009a)

13. Guillaume, R., Thierry, C., Grabot, B.: Integration of ill-known requirement into a plan. In: 39th International Conference on Computer and Industrial Engineering (CIE39), Troyes, France, July 6-8 (2009b) 